\title{
Tailoring the magnetic properties of cobalt ferrite nanoparticles using the polyol process
}

\author{
Malek Bibani ${ }^{1}$, Romain Breitwieser ${ }^{1}$, Alex Aubert ${ }^{2}$, Vincent Loyau ${ }^{2}$, Silvana Mercone ${ }^{3}$, \\ Souad Ammar ${ }^{* 1}$ and Fayna Mammeri ${ }^{* 1}$
}

Open Access

\author{
Full Research Paper \\ Address: \\ ${ }^{1}$ Université Paris Diderot, Sorbonne Paris Cité, ITODYS UMR CNRS \\ 7086, ${ }^{2}$ ENS Paris Saclay, SATIE UMR CNRS 8029, 61 Avenue du \\ Président Wilson, 94235 Cachan Cedex, France and ${ }^{3}$ Université \\ Paris Nord, Sorbonne Paris Cité, LSPM CNRS UPR-3407, 99, Av. J. \\ B. Clément, 93430 Villetaneuse, France \\ Email: \\ Souad Ammar ${ }^{*}$ - ammarmer@univ-paris-diderot.fr; Fayna Mammeri ${ }^{*}$ - \\ fayna.mammeri@univ-paris-diderot.fr \\ * Corresponding author \\ Keywords: \\ cobalt ferrite; magnetocrystalline anisotropy; magnetostriction;
} nanoparticle; non-stoichiometry; polyol process
Beilstein J. Nanotechnol. 2019, 10, 1166-1176.

doi:10.3762/bjnano.10.116

Received: 09 October 2018

Accepted: 09 May 2019

Published: 04 June 2019

This article is part of the thematic issue "Advanced hybrid nanomaterials".

Guest Editor: A. Taubert

(C) 2019 Bibani et al.; licensee Beilstein-Institut. License and terms: see end of document.

\begin{abstract}
Background: In extrinsically magnetoelectric materials made of two components, the direct magnetoelectric coupling arises from a mechanical strain transmission at the interface due to the shape change of the magnetostrictive component under an external magnetic field. Here, the size of the interface between the two components plays a crucial role. Therefore, the development of nanomaterials exhibiting large surface-to-volume ratios can help to respond to such a requirement. However, the magnetic nanoparticles (NPs) must be highly magnetostrictive and magnetically blocked at room temperature despite their nanometer-size. We describe here the use of the polyol process to synthesize cobalt ferrite $\left(\mathrm{Co}_{x} \mathrm{Fe}_{3-x} \mathrm{O}_{4}\right)$ nanoparticles with controlled size and composition and the study of the relationship between size and composition and the magnetic behavior.
\end{abstract}

Methods: We used an improved synthesis of magnetostrictive $\mathrm{Co}_{x} \mathrm{Fe}_{3-x} \mathrm{O}_{4}$ NPs based on the forced hydrolysis of metallic salts in a polyol solvent, varying the fraction $x$. Stoichiometric NPs $(x=1)$ are expected to be highly magnetostrictive while the sub-stoichiometric NPs (particularly for $x \approx 0.7$ ) are expected to be less magnetostrictive but to present a higher magnetocrystalline anisotropy constant, as previously observed in bulk cobalt ferrites. To control the size of the NPs, in order to overcome the superparamagnetic limit, as well as their chemical composition, in order to get the desired magnetomechanic properties, we carried out the reactions for two nominal precursor contents ( $x=1$ and 0.67$)$, using two different solvents, i.e., triethylene glycol (TriEG) and tetraethylene glycol (TetEG), and three different durations of refluxing (3, 6 and $15 \mathrm{~h}$ ). The structure, microstructure and composition of the resulting NPs were then investigated by using X-ray diffraction (XRD), transmission electron microscopy (TEM) and X-ray fluorescence spectroscopy (XRF), respectively. The magnetic properties were also evaluated using standard magnetometry. To measure 
the magnetostrictive response of the particles, the particles were sintered to dense pellets on which strain gauges were bonded, measuring the size variation radially, as a function of a dc magnetic field.

Findings: We found two samples, the first one being stoichiometric and magnetostrictive, and the second one being sub-stoichiometric and presenting a higher magnetization, that are appropriate to be used as ferromagnetic building blocks in nanostructured magnetoelectric materials, particularly materials based on polymers. We show that the polyol solvent and the reaction time are two key parameters to control the size and the magnetic properties of the resulting nanoparticles. We believe that these results provide relevant insights to the design of efficient magnetic and magnetostrictive nanoparticles that can be further functionalized by coupling agents, to be contacted with piezoelectric polymers.

\section{Introduction}

Recently, extrinsically (or artificially) magnetoelectric (ME) multiferroic (MF) materials have been seriously investigated for many applications in nanoelectronics [1] and energy harvesting $[2,3]$. They consist of two components, one being ferromagnetic, and the other being ferroelectric. A wide range of inorganic nanostructures, defined by their connectivity, have been prepared using different synthetic approaches. Andrew et al. published a critical viewpoint paper about the current limits of such nanostructures [4]. In these materials, the ME coupling arises from a mechanical transmission of strain originating from the shape change of the magnetostrictive component under an external magnetic field, or of the piezoelectric component under an external electrical field. Thus, the geometry of the connectivity has a huge impact on the ME efficiency and high ME coefficients are expected for extrinsic multiferroics with optimized interfaces. Despites these very enthusiastic theoretical predictions, most of the experimentally measured ME coefficients appear to be significantly smaller. This discrepancy is mainly due to the difficulties in producing hybrid materials with large and perfect interfaces [5]. The use of nanomaterials exhibiting large surface-to-volume ratios instead of bulk materials can help to overcome this limitation. To the best of our knowledge, the best improvements made in this sense were those achieved by Zheng et al., who succeeded in designing selfassembled ferromagnetic $\mathrm{CoFe}_{2} \mathrm{O}_{4}$ nanopillars embedded in a ferroelectric $\mathrm{BaTiO}_{3}$ matrix [6], and by Acevedo et al. and Liu et al., who prepared $\mathrm{CoFe}_{2} \mathrm{O}_{4}$ and $\mathrm{BaTiO}_{3}$ nanoparticles (NPs) separately and co-sintered them very quickly to avoid grain growth and coarsening [7,8]. Andrew et al. also managed to optimize and maximize the hybrid interface in polymer-based multiferroics, using $10 \mathrm{~nm}$ magnetic nanoparticles, prepared by coprecipitation and further embedded in ferroelectric polymer fibers, made by electrospinning [9]. Focusing on this latter class of materials, the polymer exhibiting the most interesting ferroand piezoelectric properties is a semi-crystalline fluoropolymer: poly(vinylidene fluoride) or PVDF. Mixing PVDF with magnetic nanoparticles leads to a higher polymer crystallinity, with NPs acting as nucleating points. Also, as established by Costa et al., the presence of these NPs promotes the crystallization of
PVDF in its $\beta$-phase, the most electroactive one, instead of its other allotropic forms [10].

Finally, another improvement consists in making the size of the ferromagnetic component as small as possible, while maintaining an efficient strain transmission (an amplitude of ca. $30 \mathrm{ppm}$ is enough for many applications [11]). Currently, the size of such nanoparticles ranges above $30 \mathrm{~nm}$ in diameter. Bulk single crystalline cobalt ferrite, for instance, exhibits a magnetostriction amplitude of 590 ppm [12] while its nanoparticle counterparts exhibit an amplitude between 90 and 215 ppm, depending, e.g., on their synthesis conditions and their composition $[13,14]$. A few years ago, Nlebedim et al. demonstrated the influence of the composition $(x)$ on the magnetocrystalline anisotropy of polycrystalline $\mathrm{Co}_{x} \mathrm{Fe}_{3-x} \mathrm{O}_{4}$. The anisotropy was found to be the highest for $x=0.7$ and 0.8 and the lowest for $x=0.2$. However, the most interesting magnetostriction effects were found at the composition of $x=1$. Therefore, the stoichiometry appears to be a key-parameter to tailor the magnetostrictive properties of cobalt ferrite materials [15].

Among the several chemical techniques that can be used for synthesizing magnetic metal-oxide NPs (such as thermal decomposition [16], hydrothermal method [17], co-precipitation of precursors [18], combustion reaction [19]), the polyol process has emerged as promising and versatile chemical route for the preparation of highly crystalline, monodisperse particles that are isotropic in shape [20,21]. Polyols act not only of solvents, but also as complexing ligands, avoiding the presence of any surfactant. Hydrolysis ratio, nature of polyol, synthesis temperature and precursor concentration are determining the final products in composition, shape, and size. Cobalt ferrite nanoparticles (NPs) have already been produced by the polyol process in one or in several steps. However, little research has focused on the relationship between the NP size and the magnetic properties and there is no literature at all regarding nonstoichiometric NPs. Artus et al. produced stoichiometric NPs of various sizes (from 2.4 to $6.2 \mathrm{~nm}$ ) depending on the hydrolysis ratio, starting from iron chloride and cobalt acetate in 1,2- 
propane-diol [22]. The blocking temperature $\left(T_{\mathrm{B}}\right)$ of the samples was found to be between $141 \mathrm{~K}$ (smallest NPs) and $315 \mathrm{~K}$ (biggest NPs). Moreover, the biggest NPs exhibited a saturation magnetization very close to that of the bulk $\left(85 \mathrm{emu} \cdot \mathrm{g}^{-1}\right.$ vs $90-95 \mathrm{emu} \cdot \mathrm{g}^{-1}$ ) indicating a very high crystallinity despite the small size of the NPs. Baldi et al. prepared stoichiometric NPs of different sizes, between 5 and $7 \mathrm{~nm}$, in diethylene glycol, starting from iron and cobalt acetates, and using a seed-mediated growth approach [23]. They obtained monodisperse and stable particles, superparamagnetic at room temperature (RT), with, once again, high saturation magnetization values for the largest ones. Hyeon et al. succeeded to produce cobalt ferrite NPs of $12 \mathrm{~nm}$ in diameter and evidenced a blocked ferromagnetic behavior for these particles at RT $\left(T_{\mathrm{B}}=320 \mathrm{~K}\right)$ [24]. They also used an etherdiol solvent as polyol during moderate heating.

Based on these former studies, sizes larger than 10-12 nm are necessary if one wants to obtain blocked cobalt ferrite particles at room temperature ( $\left.T_{\mathrm{B}}>\mathrm{RT}\right)$ [25]. At the same time, the size must be as small as possible to extend the hybrid interface in the further nanostructured hybrid ME materials and to optimize the strain transmission as well as the ME coupling.

Here, we aim to control the size of the NPs through the choice of the solvent, triethylene glycol (TriEG) and tetraethylene glycol (TetEG) with different boiling temperatures $\left(T_{\mathrm{b}}=285\right.$ and $325^{\circ} \mathrm{C}$, respectively, for TriEG and TetEG) and through the refluxing time (from 3 to $15 \mathrm{~h}$ ), assuming that a higher reaction temperature and longer reaction times will yield larger particles. In addition, we will examine different chemical compositions of the particles, i.e., the stoichiometric composition $(x=1)$ and the non-stoichiometric composition $(x=0.67)$, expecting a higher magnetostrictive coefficient for the former and a higher magnetocrystalline energy constant for the latter.

\section{Results and Discussion Structural characterization of the $\mathrm{Co}_{x} \mathrm{Fe}_{3-x} \mathrm{O}_{4}$ nanoparticles}

Nine samples have been prepared. They consist of $\mathrm{Co}_{x} \mathrm{Fe}_{3-x} \mathrm{O}_{4}$ nanoparticles distributed in two series: six of them are stoichiometric $(x=1)$ and the three others are sub-stoichiometric in cobalt $(x=0.67)$. For the first series $(x=1)$, triethylene glycol (TriEG) and tetraethylene glycol (TetEG) polyol have been used as solvents and the reaction was carried out over three different periods of time (3, 6 and $15 \mathrm{~h}$ ). For the second series $(x=0.67)$, two attempts have been made in TriEG for 3 and $6 \mathrm{~h}$, and only one in TetEG for $3 \mathrm{~h}$. The main features of all prepared compositions are collected in Table 1.

We have recorded the X-ray diffraction (XRD) patterns of all cobalt ferrite samples (Figure 1). They are all matching very well with the cubic spinel structure (ICDD no. 98-003-9131). The crystal size of each sample has been estimated through computational Rietveld refinements using MAUD software [26] (Table 1). Then, the compositions have been checked by X-ray fluorescence (XRF) experiments (Figure 2).

The crystal sizes estimated from the XRD patterns are in good agreement with the mean diameters deduced from transmission electron microscopy (TEM) images (Table 1), meaning that the nanoparticles are monocrystalline. The micrographs given in Figure 3 and Figure 4 present the two series of nanoparticles ( $x=1$ and $x=0.67$ ) as a function of the polyol and as a function of the reaction time. All of them appear to be quite uniform in size since the standard deviations do not exceed $20 \%$ of the average diameters. The particle size histograms presented in Figure 3 and Figure 4 have been made applying Sturges' rule [27]. They were then fitted using a log-normal function (Equation 1) and the median diameter $D$ as well as the dispersion $\sigma$ were determined (see Table 1).

Table 1: Main structural features of the $\mathrm{Co}_{x} \mathrm{Fe}_{3-x} \mathrm{O}_{4}$ nanoparticles, prepared by the polyol process

\begin{tabular}{|c|c|c|c|c|c|c|}
\hline sample & $(x)$ & polyol & reaction time & $\begin{array}{l}d(\mathrm{~nm}) \\
\text { from XRD }\end{array}$ & $\begin{array}{l}d(\mathrm{~nm}) \\
\text { from TEM }\end{array}$ & $\begin{array}{l}\text { cell parameter } \\
(\AA) \text { from XRD }\end{array}$ \\
\hline Co-1-TriEG-3 & 1 & TriEG & 3 & $7 \pm 1$ & $5.6 \pm 0.2$ & $8.404 \pm 0.002$ \\
\hline Co-1-TriEG-6 & 1 & TriEG & 6 & $8 \pm 1$ & $6.7 \pm 0.2$ & $8.401 \pm 0.002$ \\
\hline Co-1-TriEG-15 & 1 & TriEG & 15 & $9 \pm 1$ & $8.4 \pm 0.2$ & $8.402 \pm 0.002$ \\
\hline Co-1-TetEG-3 & 1 & TetEG & 3 & $8 \pm 1$ & $7.8 \pm 0.3$ & $8.405 \pm 0.002$ \\
\hline Co-1-TetEG-6 & 1 & TetEG & 6 & $10 \pm 1$ & $9.6 \pm 0.2$ & $8.404 \pm 0.002$ \\
\hline Co-1-TetEG-15a & 1 & TetEG & 15 & $13 \pm 1$ & $12.0 \pm 0.3$ & $8.399 \pm 0.002$ \\
\hline Co-0.67-TriEG-3 & 0.67 & TriEG & 3 & $12 \pm 1$ & $10.2 \pm 0.2$ & $8.397 \pm 0.002$ \\
\hline Co-0.67-TriEG-6 & 0.67 & TriEG & 6 & $13 \pm 1$ & $12.1 \pm 0.2$ & $8.397 \pm 0.002$ \\
\hline Co-0.67-TetEG-3 & 0.67 & TetEG & 3 & $13 \pm 1$ & $12.0 \pm 0.3$ & $8.398 \pm 0.002$ \\
\hline
\end{tabular}

${ }^{\mathrm{a} C o-1-T e t E G-15}$ shows traces of metallic $\mathrm{Co}$. 


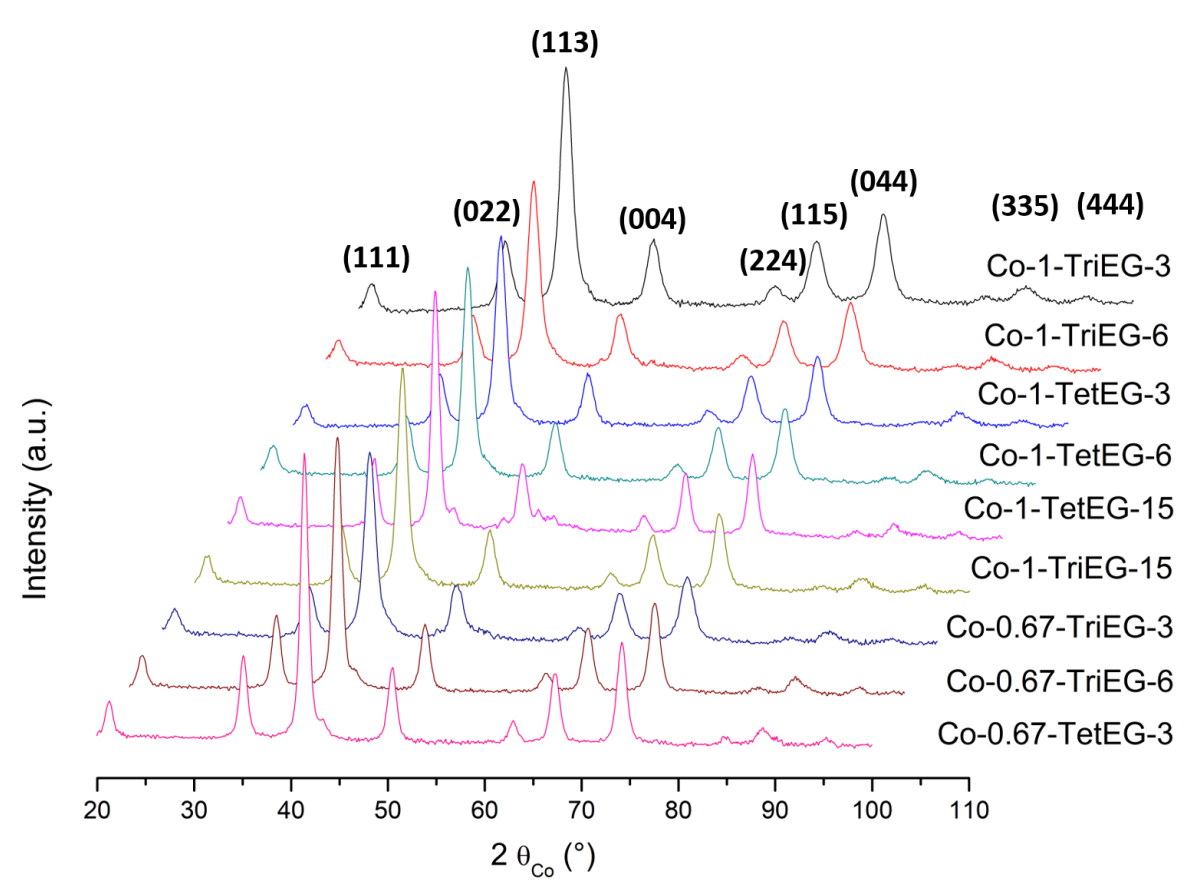

Figure 1: XRD patterns of all the produced $\mathrm{Co}_{x} \mathrm{Fe}_{3-x} \mathrm{O}_{4}$ powders.

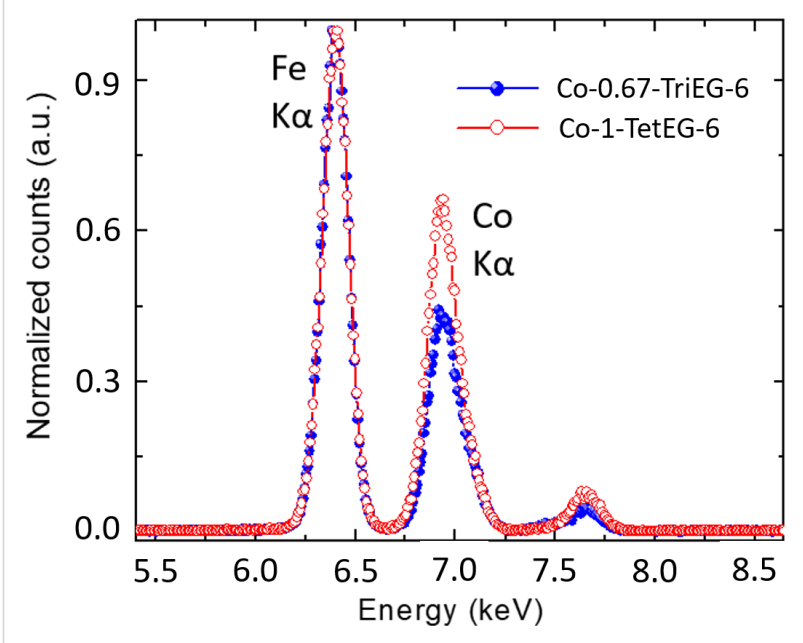

Figure 2: X-ray fluorescence experiments performed on two representative samples, Co-1-TetEG-6 and Co-0.67-TriEG-6.

$$
f(D)=\frac{1}{\sqrt{2 \pi \sigma D}} \times \exp \left[\frac{\ln ^{2}\left(\frac{D}{D_{0}}\right)}{2 \sigma^{2}}\right]
$$

Then, the mean diameter $\left\langle D>\right.$ and standard deviation $\sigma_{D}$ were calculated (Equation 2 and Equation 3).

$$
\langle D\rangle=D_{0} \times \exp \left(\frac{\sigma^{2}}{2}\right)
$$

$$
\sigma_{D}=\langle D\rangle \times\left[\exp \left(\sigma^{2}-1\right)\right]^{1 / 2}
$$

\section{Influence of the time of synthesis}

The reactions were carried out for different periods of time: 3,6 and $15 \mathrm{~h}$. Table 1 shows unambiguously that the NP diameter is increasing when the duration of the reaction increases, when the other conditions are the same.

\section{Influence of the solvent}

We used two polyols: TriEG and TetEG (Figure 4). The former presents a shorter backbone and a higher dielectric constant $\left(\varepsilon_{\mathrm{r}(\mathrm{TriEG})}=23.7\right.$ vs $\left.\varepsilon_{\mathrm{r}(\mathrm{TetEG})}=20.4\right)$ while the latter exhibits a larger molecular weight and is assumed to be a little more polar $\left(\mu_{\mathrm{TetEG}}=5.84 \mathrm{D}\right.$ vs $\left.\mu_{\mathrm{TriEG}}=5.58 \mathrm{D}\right)$ [28]. Regardless of reaction time and composition, we observe that the NP diameter is higher when tetraethylene glycol is used instead of triethylene glycol. Dipolar moment and dielectric constant of the two molecules are very similar and we can assume that they both exhibit the same strength to dissolve the ionic precursors. TetEG has a longer backbone than TriEG and can chelate bigger colloidal species, which may promote the growth better than TriEG. Another parameter may contribute to explain this size difference between the TriEG- and the TetEG-derived particles, i.e., the reaction temperature and the boiling points of the polyols. Indeed, the refluxing temperature was observed to be lower for TriEG but not so much regarding the boiling points of the two polyols. Considering the very probable hypothesis that particle 


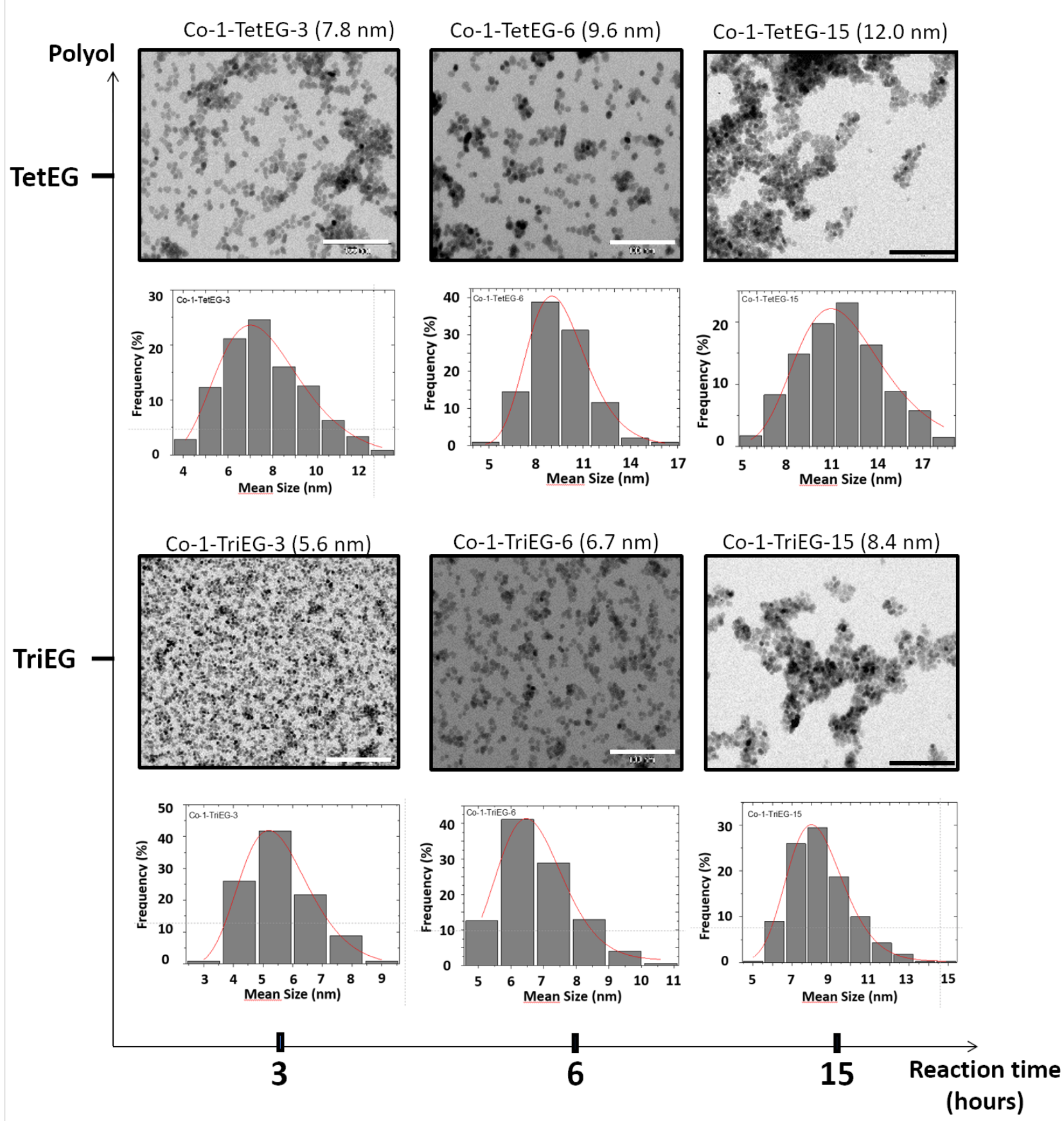

Figure 3: TEM images of $\mathrm{CoFe}_{2} \mathrm{O}_{4} \mathrm{NPs}$ as a function of the polyol nature and the reaction time, and the corresponding diameter distributions and lognormal fits. Scale bar $=100 \mathrm{~nm}$.

nucleation proceeds when the boiling temperature of the reaction medium is reached (leading to the lowest viscosity), one can expect the formation of much more nuclei when the reaction temperature is close to the boiling point of the solvent [29]. This was the case when TriEG was used. Thus, the crystal growth by solute diffusion occurred on a larger number of nuclei, leading to a smaller final particle size: In contrast, a smaller number of nuclei was produced in TetEG, since the reaction temperature was considerably lower than the boiling point.

\section{Influence of the starting stoichiometry}

Two chemical compositions of $\mathrm{Co}_{x} \mathrm{Fe}_{3-x} \mathrm{O}_{4}$ NPs have been prepared: $x=1$ and $x=0.67$. We observe that, for the same polyol used and the same time of reaction, the sub-stoichiometric nanoparticles are always bigger by at least $5 \mathrm{~nm}$ than the stoi- 


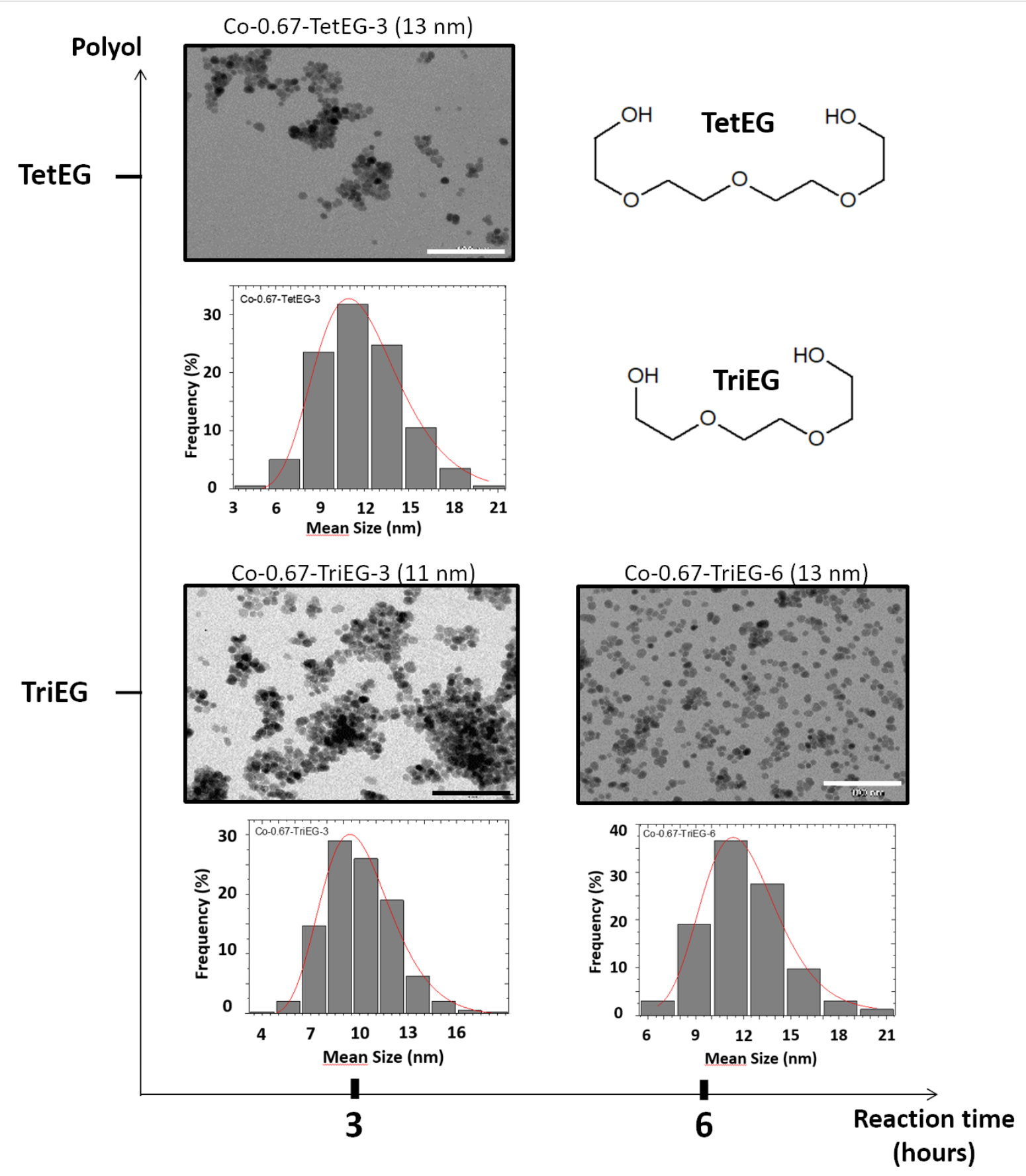

Figure 4: TEM images of $\mathrm{Co}_{0.67} \mathrm{Fe}_{2.33} \mathrm{O}_{4} \mathrm{NPs}$ as a function of the polyol nature and the reaction time, and the corresponding diameter distributions and log-normal fits. Scale bar $=100 \mathrm{~nm}$. The formulas of the polyols are given in the insert.

chiometric ones. The dependence of the NP size on $x$ is still poorly understood, and it would be interesting to investigate it. But at this stage of our study we only noticed it, with the aim of elucidating it in further experiments.

\section{Aggregation}

From the XRD and TEM measurements, we have deduced the average diameter of the produced particles, assuming them to be almost spherical single crystals uniform in size. Moreover, from TEM images, we can evaluate the morphology developed by the NPs. In the stoichiometric samples, the nanoparticles obtained after $15 \mathrm{~h}$ of reaction are clearly more aggregated than those obtained after $6 \mathrm{~h}$, most likely due to stronger van der Waals and/ or magnetostatic interactions between bigger nanoparticles (promoted by the drying of the NPs during the sample preparation for TEM) resulting in the clustering of particles. This ob- 
servation has been made after syntheses with TetEG and TriEG, and after drop casting the same quantity of NPs under the same conditions. In the sub-stoichiometric series, Co-0.67-TriEG-3 NPs exhibit the highest degree of aggregation.

\section{Magnetic properties}

Standard magnetometry has been carried out on all $\mathrm{Co}_{x} \mathrm{Fe}_{3-x} \mathrm{O}_{4}$ NPs with a special emphasis on the biggest NPs, for which a blocked ferromagnetic behavior is expected at RT $\left(T_{\mathrm{B}}>\mathrm{RT}\right)$.

The zero-field-cooled (ZFC) and field-cooled (FC) magnetization as a function of the temperature is shown in Figure 5. In general, the recorded magnetic behavior is that of ferrite particles in their single magnetic domain state. As it is summarized in Table 2, quite all the samples showed very high $T_{\mathrm{B}}$ values $(>300 \mathrm{~K})$. The only superparamagnetic nanoparticles at room temperature are the stoichiometric particles synthesized in TriEG for $6 \mathrm{~h}$ (Co-1-TriEG-6). In this case, $T_{\mathrm{B}}$ was found to be equal to $275 \mathrm{~K}$; although the saturation magnetization value is

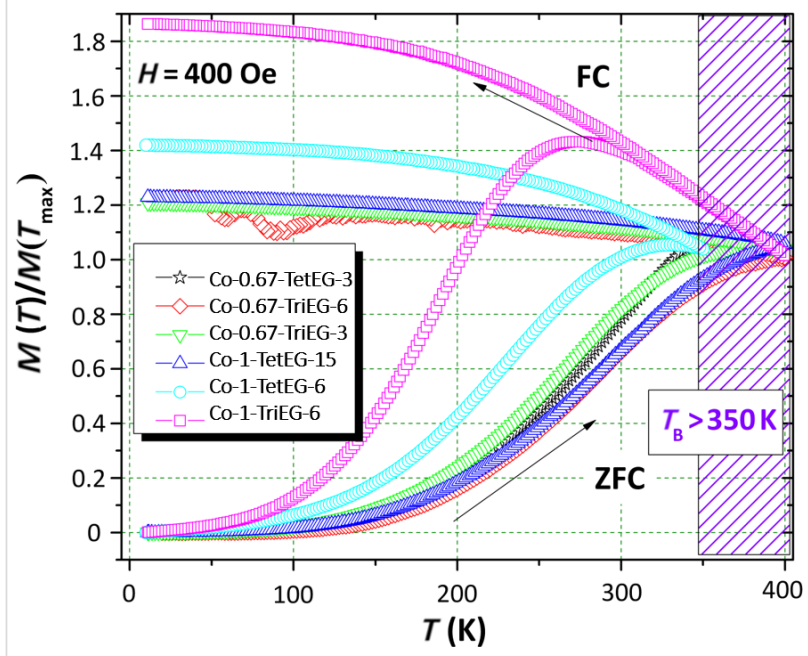

Figure 5: Thermal variation of the normalized DC magnetic magnetization measured in ZFC and FC conditions. pretty good for this composition at the nanoscale level $(d=8 \mathrm{~nm})$, the superparamagnetic behavior observed at room temperature is not suitable for the targeted applications. Interestingly, such high $T_{\mathrm{B}}$ values confirm the high crystalline quality of the produced NPs. We pursued our investigations by plotting the variation of the magnetization of these particles as a function of the magnetic field at RT, typically $T=300 \mathrm{~K}$ (Figure 6). Low-temperature $(T=10 \mathrm{~K})$ hysteresis behavior is not reported, as it is comparable to that at the RT; however, the coercive fields that have been measured at this temperature are summarized in Table 2, as well as those obtained at RT.

Higher values of coercivity have been observed for NPs synthesized in TetEG. Again, extending the reaction time in TriEG up to $6 \mathrm{~h}$ does not yield the high coercivity observed in TetEG at 300 and $10 \mathrm{~K}$. Thus, for equal nanoparticle sizes, equal time of reaction and equal composition, the TetEG solvent seems to optimize the magnetic behavior of the nanopowders in regard to the targeted applications. Additionally, they present saturation magnetization values among the highest that can be found in the literature for this particle size [30,31].

Based on all these structural and magnetic results, we chose to focus on the following two samples: Co-1-TetEG-6 and Co-0.67-TriEG-6. As magnetostriction measurement requires bulk samples, the samples were sintered by using spark plasma sintering. We are aware that the measured magnetostrictive coefficients on the prepared pellets do not correspond exactly to those of the bare particles, but they are quite indicative of the magnetostrictive behavior of the starting powders. Magnetostriction measurements were performed by applying an in-plane external magnetic field (see the Experimental section). The results are presented in Figure 7.

For the Co-0.67-TriEG-6 consolidated derivative, a maximal radial magnetostrictive deformation $\left(L-L_{0}\right) / L_{0}$ of $-76 \mathrm{ppm}$ has been observed for the demagnetized state, when the magnetic field is applied for the first time ( $L_{0}$ is the initial length of the material with $H=0$ ). After the in-plane saturation of the magne-

\begin{tabular}{lllll} 
Table 2: Blocking temperature $\left(T_{\mathrm{B}}\right)$, saturation magnetization $\left(M_{\mathrm{S}}\right)$ and coercive field $\left(H_{\mathrm{C}}\right)$ of the $\mathrm{Co}_{\mathrm{X}} \mathrm{Fe}_{3-\mathrm{x}} \mathrm{O}_{4}$ nanoparticles. \\
& $T_{\mathrm{B}}(\mathrm{K})$ & $M_{\mathrm{S}}\left(\mathrm{emu} \cdot \mathrm{g}^{-1}\right)$ at $300 \mathrm{~K}$ & $H_{\mathrm{C}}(\mathrm{Oe})$ at $300 \mathrm{~K}$ & $H_{\mathrm{C}}(\mathrm{Oe})$ at $10 \mathrm{~K}$ \\
\hline sample & ca. 275 & 62 & 28 & 690 \\
Co-1-TriEG-6 & ca. 330 & 51 & 94 & 10041 \\
Co-1-TetEG-6 & $>350$ & 67 & 220 & 13300 \\
Co-0.67-TriEG-3 & $>350$ & 71 & 60 & 8000 \\
Co-0.67-TriEG-6 & $>350$ & 77 & 220 & 780 \\
Co-0.67-TetEG-3 & $>350$ & 104 & 300 & 13700 \\
\hline
\end{tabular}

${ }^{\mathrm{a} C o-1-T e t E G-15}$ shows traces of metallic Co. 


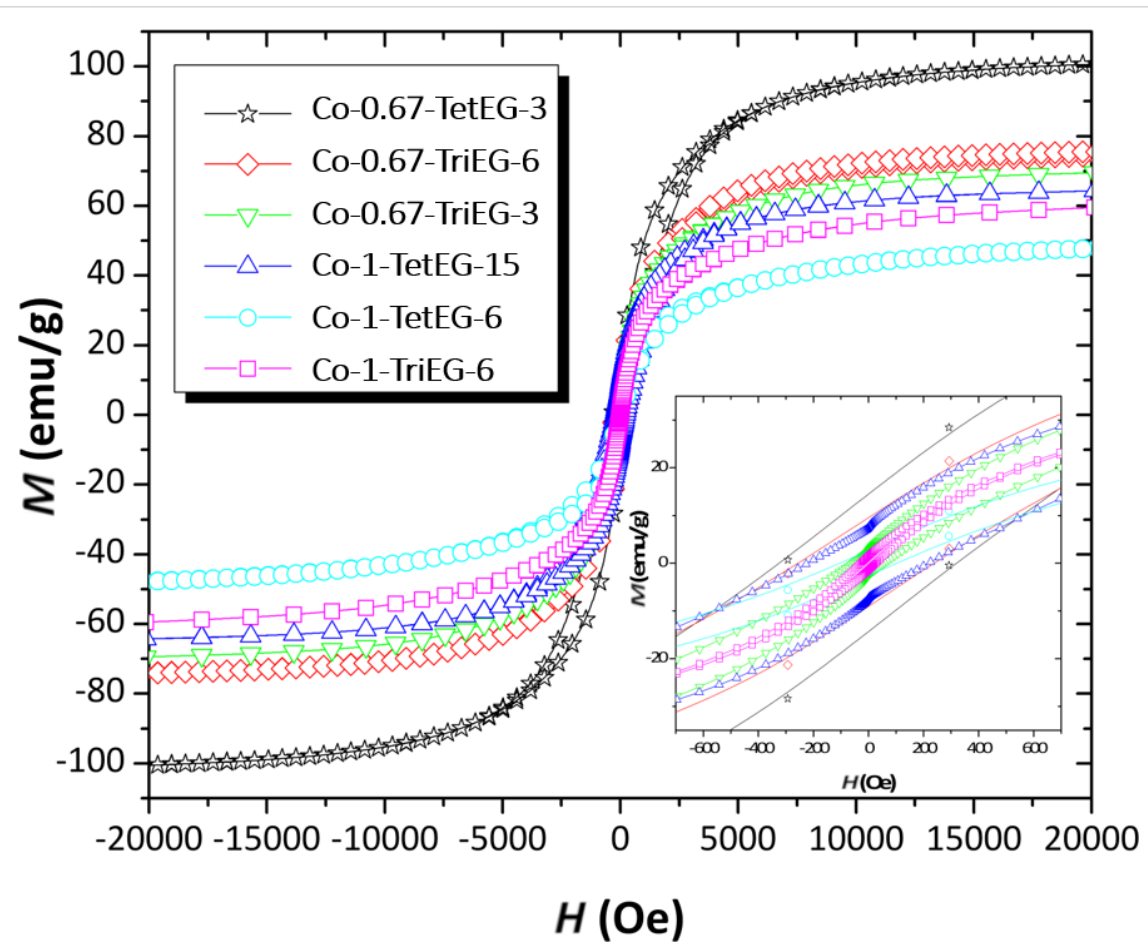

Figure 6: Magnetisation curves of NPs measured at $300 \mathrm{~K}$. Inset: zoom-in of the coercive behavior.
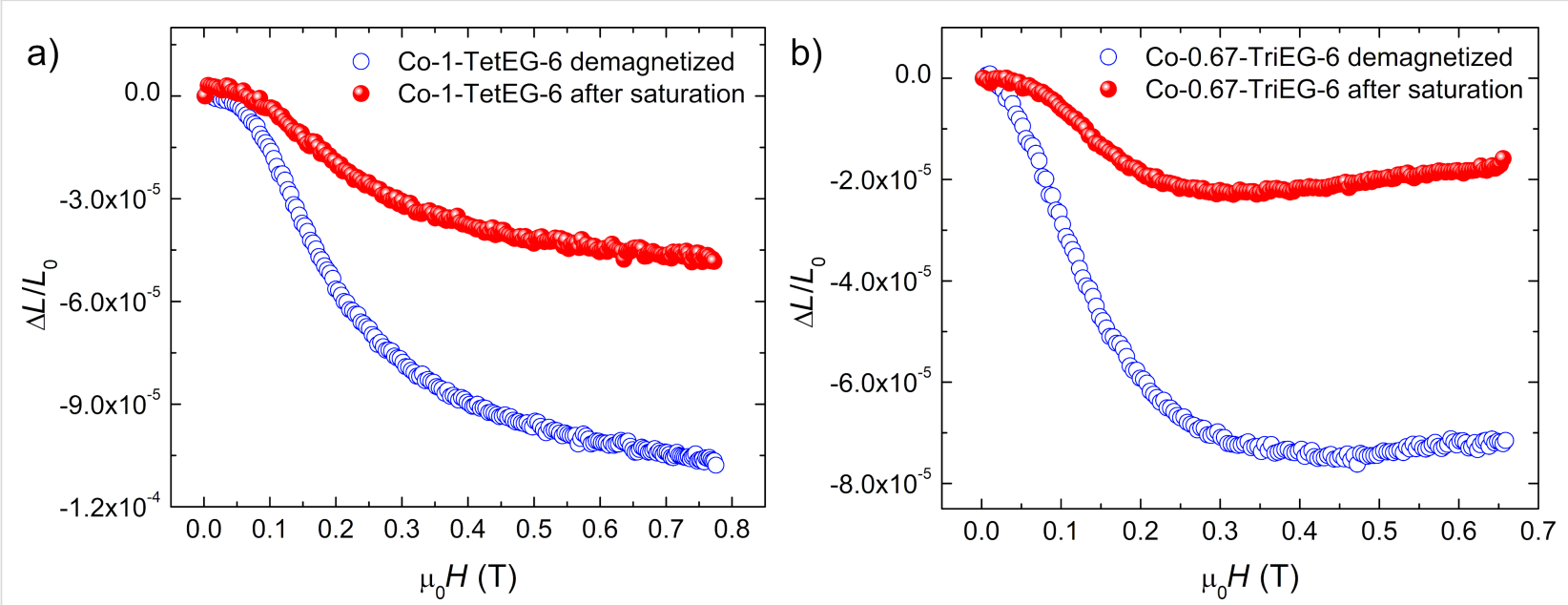

Figure 7: Radial magnetostriction as a function of the applied magnetic field for a) Co-1-TetEG-6 and b) Co-0.67-TriEG-6 consolidated derivatives with measurements carried out from either the demagnetized (blue circles) or the in-plane saturated (red circles) state.

tization, a second measurement has been carried out showing a $\Delta L / L_{0}$ coefficient strongly reduced to $-23 \mathrm{ppm}$ due to the remanence effect of the magnetization. To recover the magnetostriction measured during the first cycle, it is necessary to preliminary demagnetize the sample or to saturate the magnetization along a transversal direction.

For the Co-1-TetEG-6 consolidated derivative, the highest magnetostrictive coefficient of $-106 \mathrm{ppm}$ has been obtained from the demagnetized state while the second cycle of measurements indicated a deformation of $-47 \mathrm{ppm}$. We therefore observed that the magnetic history has similar effects on the magnetostrictive response of both samples. Moreover, reducing the Co amount in the initial powders by ca. $30 \%$ leads to a loss of ca. $30 \%$ of the magnetostrictive response. Such a result is quite reasonable since the magnetostriction of cobalt ferrites is induced by $\mathrm{Co}^{2+}$ ions in octahedral sites and the spin-orbit-lattice interaction with the distorted cubic crystal 
field. The other striking conclusion is that the larger deformation of stoichiometric cobalt ferrites nanoparticles under magnetic field makes them more promising to enhance the ME properties of nanomaterials.

\section{Conclusion}

We have described here the synthesis procedures of magnetostrictive $\mathrm{Co}_{x} \mathrm{Fe}_{3-x} \mathrm{O}_{4}(x=1$ and $x=0.67) \mathrm{NPs}$, using the polyol process. The produced NPs have been well characterized using X-ray techniques (diffraction and fluorescence) and transmission electron microscopy. Most of the NPs are above $10 \mathrm{~nm}$ in size, magnetically stable and blocked at room temperature $\left(T_{\mathrm{B}}>\mathrm{RT}\right)$. Moreover, they exhibit saturation magnetization values among the highest presented in literature for their typical size. For the syntheses, we used two different polyol solvents, TriEG and TetEG and carried out the reactions for three different periods of time (3, 6 and $15 \mathrm{~h}$ ). We could identify two samples, the first one being stoichiometric and the second being sub-stoichiometric, appropriate for the use as ferromagnetic building blocks in nanostructured magnetoelectric materials, particularly polymer-based hybrid materials. We hope this work is providing some insight into the ability to design efficient magnetic and magnetostrictive particles that can be further functionalized by coupling agents such as phosphonic acids to be introduced in polymers $[32,33]$.

\section{Experimental \\ Synthesis of the nanoparticles}

The synthesis of the $\mathrm{Co}_{x} \mathrm{Fe}_{3-x} \mathrm{O}_{4}$ nanoparticles (NPs) was carried out using the polyol process [22], starting from iron and cobalt acetates, $\mathrm{Fe}\left(\mathrm{CH}_{3} \mathrm{COO}\right)_{2}$ and $\mathrm{Co}\left(\mathrm{CH}_{3} \mathrm{COO}\right)_{2} \cdot 4 \mathrm{H}_{2} \mathrm{O}$ (Acros and Aldrich, respectively) in two nominal ratios ( $x=1$ or $x=0.67$ ) [34]. We used two different polyols, TriEG and TetEG. The reaction mixture was heated up to reflux (270 or $290{ }^{\circ} \mathrm{C}$, depending on the solvent) and maintained under reflux for 3, 6 or $15 \mathrm{~h}$ to obtain single-phase NPs of various sizes. After being cooled to RT, the black nanoparticle powders were recovered by several centrifugation cycles and washing with acetone. At the end, they were dried overnight in air at $50{ }^{\circ} \mathrm{C}$. Table 1 is collecting all the main features of these samples.

\section{Characterization of the nanoparticles}

\section{Structure}

The XRD patterns of the recovered powders have been recorded on an X'Pert Pro PANanalytical diffractometer (Co Ka radiation), in the range of $10-100^{\circ}(2 \theta)$ with a scan step of $0.02^{\circ}$. The morphology of the NPs has been determined by TEM observations, using a JEOL-100 CX II microscope operating at $100 \mathrm{kV}$. The mean diameter and standard deviation were inferred from image analysis of ca. 350-400 particles using ImageJ software and correlated to the microstructural information (mainly crystal size and micro-strain-induced lattice deformation) inferred from Rietveld analysis of the XRD data using MAUD software [26]. The chemical analysis of the particles was checked by XRF, using a Panalytical MINIPAL4 $\mathrm{X}$-ray fluorescence spectrometer, equipped with a rhodium $\mathrm{X}$-ray tube operating at $30 \mathrm{kV}$ and $87 \mu \mathrm{A}$ current emission. Quantification was determined from pre-plotted calibration curves using standard Co and Fe solutions.

\section{Magnetic properties}

Direct-current magnetic measurements were performed using a Quantum Design MPMS 3 superconducting quantum interference device working as a vibrational standard magnetometer. The thermal variation of the magnetic susceptibility $\chi(T)$ were recorded in both $\mathrm{ZFC}$ and FC modes, in the temperature range of $10-400 \mathrm{~K}$ under a magnetic field of $400 \mathrm{Oe}$. The magnetization as a function of the magnetic field $M(H)$ was also recorded at low temperature $(10 \mathrm{~K})$ and room temperature $(300 \mathrm{~K})$ cycling the magnetic field between $-70 \mathrm{kOe}$ and $+70 \mathrm{kOe}$. A sampling tube made from a specific pod from Quantum Design has been used to mechanically block the analyzed powders (few milligrams) during the measurements.

\section{Magnetostriction}

Selected particles have been first consolidated into dense pellets by using spark plasma sintering, applying a uniaxial pressure of $100 \mathrm{MPa}$ and heating the pressed powder up to $500{ }^{\circ} \mathrm{C}$ in a graphite die (more details are given in [35]). Then, a resistive strain gauge (EA-06-062TT-120, Micro-Measurements) was glued (with epoxy resin) on the top face of each polished pellet, to perform extensometry measurements in presence of a longitudinally applied magnetic field $H$ (Figure $8 \mathrm{~b}$ ). The in-plane direction of $H$ has been chosen to avoid demagnetizing field effects occurring in the out-of-plane direction, which is known to affect the ME response. Then, the relative strain $\left(L-L_{0}\right) / L_{0}$ of each pellet was deduced from the measurement of the resistance relative variation $\left(R-R_{0}\right) / R_{0}$ of the gauge following: $\left(L-L_{0}\right) / L=(1 / K) .\left(R-R_{0}\right) / R_{0}$, where $K$ is the gauge factor ( $K=2), R_{0}=120 \Omega$ is the initial unstrained resistance of the gauge, and $L_{0}$ is the unstrained length of the active part of the gauge. The sample has been placed into an electromagnet, and a magnetic field varying from 0 up to $1 \mathrm{~T}$ has been applied. This is sufficient to reach the $\mathrm{Co}_{x} \mathrm{Fe}_{3-x} \mathrm{O}_{4}$ saturation magnetization (generally, $\mu_{0} H_{\text {sat }}<0.5 \mathrm{~T}$ at $\mathrm{RT}$ ). In our measurements, $\left(L-L_{0}\right) / L$ coincides with the magnetostriction coefficient $\lambda_{11}$ because the strain measurement is done along the direction of the applied field $H$. For magnetoelectric applications, one should note that the transverse magnetostrictive coefficient $\left(\lambda_{21}\right)$ is also of interest. In case of isotropic samples, the transversal coefficient is expected to be half the longitudinal one and opposite in sign [36]. 


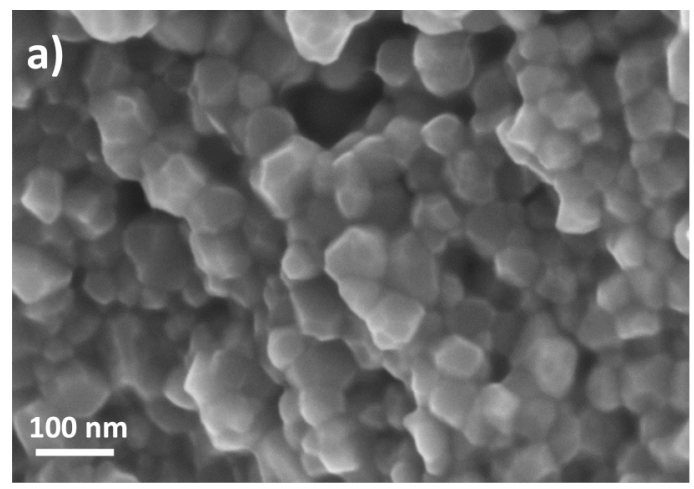

b)

$H$ in plane

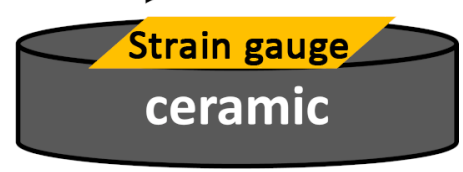

Figure 8: a) Representative scanning electron microscopy (SEM) image of the cobalt ferrite consolidated derivative and b) a schematic illustration of the custom-made magnetostriction measurement setup.

Using this procedure, we have evaluated the magnetostrictive properties of the two most interesting samples, i.e., $\mathrm{Co}_{0.67} \mathrm{Fe}_{2.33} \mathrm{O}_{4}$, known to exhibit the highest magnetocrystalline anisotropy (Co-0.67-TriEG-6), and $\mathrm{CoFe}_{2} \mathrm{O}_{4}$, known to present the highest magnetostriction (Co-1-TetEG-6).

\section{Acknowledgements}

We would like to thank M. Ludovic Mouton and Dr. Sophie Nowak (Université Paris Diderot) for their technical support on TEM and XRF analysis, respectively. We would also like to thank Prof. Frédéric Mazaleyrat (ENS Paris-Saclay) for the fruitful discussions on nanomagnetism. The magnetic measurements performed by Dr. Silvana Mercone at ESPCI have been supported through grants from the Ile-de-France Regional Council. ANR (Agence Nationale de la Recherche) and CGI (Commissariat à l'Investissement d'Avenir) are gratefully acknowledged for their financial support of this work through Labex SEAM (Science and Engineering for Advanced Materials and devices), ANR 11 LBX 086, ANR 11 IDEX 0502 ANR is also deeply acknowledged for the financial support to MULTIFERROFLEX Young Researcher project (ANR-14CE08-0005-01) attributed to FM.

\section{ORCID ${ }^{\circledR}$ iDs}

Malek Bibani - https://orcid.org/0000-0002-0950-0875 Alex Aubert - https://orcid.org/0000-0002-0816-4566 Silvana Mercone - https://orcid.org/0000-0002-5790-1032 Souad Ammar - https://orcid.org/0000-0002-1656-0016

\section{References}

1. Chu, Y.-H.; Martin, L. W.; Holcomb, M. B.; Gajek, M.; Han, S.-J.; He, Q.; Balke, N.; Yang, C.-H.; Lee, D.; Hu, W.; Zhan, Q.; Yang, P.-L.; Fraile-Rodríguez, A.; Scholl, A.; Wang, S. X.; Ramesh, R. Nat. Mater. 2008, 7, 478-482. doi:10.1038/nmat2184

2. Wan, C.; Bowen, C. R. J. Mater. Chem. A 2017, 5, 3091-3128. doi:10.1039/c6ta09590a
3. Onuta, T.-D.; Wang, Y.; Long, C. J.; Takeuchi, I. Appl. Phys. Lett. 2011, 99, 203506. doi:10.1063/1.3662037

4. Andrew, J. S.; Starr, J. D.; Budi, M. A. K. Scr. Mater. 2014, 74, 38-43. doi:10.1016/j.scriptamat.2013.09.023

5. Liu, R.; Zhao, Y.; Huang, R.; Zhao, Y.; Zhou, H. J. Mater. Chem. 2010, 20, 10665-10670. doi:10.1039/c0jm02602f

6. Zheng, H.; Wang, J.; Lofland, S.; Ma, Z.; Mohaddes-Ardabili, L.; Zhao, T.; Salamanca-Riba, L.; Shinde, S.; Ogale, S.; Bai, F.; Viehland, D.; Jia, Y.; Schlom, D.; Wuttig, M.; Roytburd, A.; Ramesh, R. Science 2004, 303, 661-663. doi:10.1126/science.1094207

7. Acevedo, U.; Lopez-Noda, R.; Breitwieser, R.; Calderon, F.; Ammar, S.; Valenzuela, R. AIP Adv. 2017, 7, 055813. doi:10.1063/1.4974493

8. Liu, Y.; Xu, G.; Xie, Y.; Lv, H.; Huang, C.; Chen, Y.; Tong, Z.; Shi, J.; Xiong, R. Ceram. Int. 2018, 44, 9649-9655. doi:10.1016/j.ceramint.2018.02.192

9. Andrew, J. S.; Clarke, D. R. Langmuir 2008, 24, 8435-8438. doi:10.1021/la801617q

10. Martins, P.; Costa, C. M.; Benelmekki, M.; Botelho, G.; Lanceros-Méndez, S. J. Mater. Sci. 2013, 48, 2681-2689. doi:10.1007/s10853-012-7063-1

11. Affanni, A.; Guerra, A.; Dallagiovanna, L.; Chiorboli, G. Design and characterization of magnetostrictive linear displacement sensors. In Proceedings of the 21st IEEE Instrumentation and Measurement Technology Conference (IEEE Cat. No.04CH37510), IEEE Publishing: Piscataway, NJ, U.S.A., 2004; pp 206-209. doi:10.1109/imtc.2004.1351029

12. Bozorth, R. M.; Tilden, E. F.; Williams, A. J. Phys. Rev. 1955, 99 , 1788-1798. doi:10.1103/physrev.99.1788

13. Nlebedim, I. C.; Ranvah, N.; Williams, P. I.; Melikhov, Y.; Snyder, J. E.; Moses, A. J.; Jiles, D. C. J. Magn. Magn. Mater. 2010, 322, 1929-1933. doi:10.1016/j.jmmm.2010.01.009

14. Nlebedim, I. C.; Snyder, J. E.; Moses, A. J.; Jiles, D. C. J. Magn. Magn. Mater. 2010, 322, 3938-3942. doi:10.1016/j.jmmm.2010.08.026

15. Nlebedim, I. C.; Snyder, J. E.; Moses, A. J.; Jiles, D. C. IEEE Trans. Magn. 2012, 48, 3084-3087. doi:10.1109/tmag.2012.2200884

16. Herrera, A. P.; Polo-Corrales, L.; Chavez, E.; Cabarcas-Bolivar, J.; Uwakweh, O. N. C.; Rinaldi, C. J. Magn. Magn. Mater. 2013, 328, 41-52. doi:10.1016/j.jmmm.2012.09.069 
17. Repko, A.; Vejpravová, J.; Vacková, T.; Zákutná, D.; Nižňanský, D. J. Magn. Magn. Mater. 2015, 390, 142-151.

doi:10.1016/j.jmmm.2015.04.090

18. Soler, M. A. G.; Lima, E. C. D.; da Silva, S. W.; Melo, T. F. O.; Pimenta, A. C. M.; Sinnecker, J. P.; Azevedo, R. B.; Garg, V. K.; Oliveira, A. C.; Novak, M. A.; Morais, P. C. Langmuir 2007, 23, 9611-9617. doi:10.1021/la701358g

19. Nakagomi, F.; da Silva, S. W.; Garg, V. K.; Oliveira, A. C.; Morais, P. C.; Franco Júnior, A.; Lima, E. C. D. J. Appl. Phys. 2007, 101, 09M514. doi:10.1063/1.2712821

20. Ammar, S.; Helfen, A.; Jouini, N.; Fiévet, F.; Rosenman, I.; Villain, F.; Molinié, P.; Danot, M. J. Mater. Chem. 2001, 11, 186-192. doi:10.1039/b003193n

21. Mammeri, F.; Mamèche, F.; Kataya, Z.; Yaacoub, N.; Slawska-Waniewska, A.; Menguy, N.; Grenèche, J.-M.; Ammar, S. MRS Online Proc. Libr. 2011, 1359, No. mrss11-1359-nn08-07. doi:10.1557/opl.2011.883

22. Artus, M.; Ben Tahar, L.; Herbst, F.; Smiri, L.; Villain, F.; Yaacoub, N.; Grenèche, J.-M.; Ammar, S.; Fiévet, F. J. Phys.: Condens. Matter 2011, 23, 506001. doi:10.1088/0953-8984/23/50/506001

23. Baldi, G.; Bonacchi, D.; Innocenti, C.; Lorenzi, G.; Sangregorio, C. J. Magn. Magn. Mater. 2007, 311, 10-16. doi:10.1016/j.jmmm.2006.11.157

24. Hyeon, T. Chem. Commun. 2003, 8, 927-934. doi:10.1039/b207789b

25. Dai, Q.; Nelson, A. Chem. Soc. Rev. 2010, 39, 4057-4066. doi:10.1039/b812669k

26. Lutterotti, L.; Matthies, S.; Wenk, H. R. IUCr: Newsletter of the CPD 1999, 21, 14-15.

27. Aragón, F. H.; Coaquira, J. A. H.; Villegas-Lelovsky, L.; da Silva, S. W.; Cesar, D. F.; Nagamine, L. C. C. M.; Cohen, R.;

Menéndez-Proupin, E.; Morais, P. C. J. Phys.: Condens. Matter 2015, 27, 095301. doi:10.1088/0953-8984/27/9/095301

28. Fiévet, F.; Ammar-Merah, S.; Brayner, R.; Chau, F.; Giraud, M.; Mammeri, F.; Peron, J.; Piquemal, J.-Y.; Sicard, L.; Viau, G. Chem. Soc. Rev. 2018, 47, 5187-5233. doi:10.1039/c7cs00777a

29. Bailon, J. J.; Dorlot, L. M. Des matériaux; Ecole Polytechnique de Montréal: Québec, Canada, 1986.

30. Lu, R. E.; Chang, K. G.; Fu, B.; Shen, Y. J.; Xu, M. W.; Yang, S.; Song, X. P.; Liu, M.; Yang, Y. D. J. Mater. Chem. C 2014, 2, 8578-8584. doi:10.1039/c4tc01415d

31. Ajroudi, L.; Mliki, N.; Bessais, L.; Madigou, V.; Villain, S.; Leroux, C. Mater. Res. Bull. 2014, 59, 49-58. doi:10.1016/j.materresbull.2014.06.029

32. Ourry, L.; Mammeri, F.; Toulemon, D.; Gaudisson, T.; Delamar, M.; Ammar, S. RSC Adv. 2016, 6, 49973-49979. doi:10.1039/c6ra06963k

33. Ourry, L.; Toulemon, D.; Ammar, S.; Mammeri, F. Beilstein J. Nanotechnol. 2017, 8, 408-417. doi:10.3762/bjnano.8.43

34. Artus, M.; Ammar, S.; Sicard, L.; Piquemal, J.-Y.; Herbst, F.; Vaulay, M.-J.; Fiévet, F.; Richard, V. Chem. Mater. 2008, 20, 4861-4872. doi:10.1021/cm702464e

35. Gaudisson, T.; Artus, M.; Acevedo, U.; Herbst, F.; Nowak, S.; Valenzuela, R.; Ammar, S. J. Magn. Magn. Mater. 2014, 370, 87-95. doi:10.1016/j.jmmm.2014.06.014

36. Aubert, A.; Loyau, V.; Mazaleyrat, F.; LoBue, M. J. Eur. Ceram. Soc. 2017, 37, 3101-3105. doi:10.1016/j.jeurceramsoc.2017.03.036

\section{License and Terms}

This is an Open Access article under the terms of the Creative Commons Attribution License (http://creativecommons.org/licenses/by/4.0). Please note that the reuse, redistribution and reproduction in particular requires that the authors and source are credited.

The license is subject to the Beilstein Journal of Nanotechnology terms and conditions: (https://www.beilstein-journals.org/bjnano)

The definitive version of this article is the electronic one which can be found at:

doi:10.3762/bjnano.10.116 